\title{
miR-517a is an independent prognostic marker and contributes to cell migration and invasion in human colorectal cancer
}

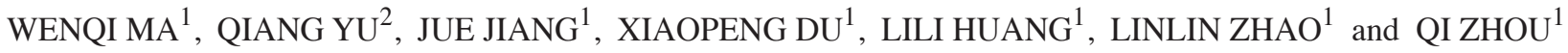 \\ Departments of ${ }^{1}$ Ultrasound and ${ }^{2}$ Pediatric Surgery, The Second Affiliated Hospital of Xi'an Jiaotong University, \\ Xi'an, Shaanxi 710004, P.R. China
}

Received January 11, 2015; Accepted February 12, 2016

DOI: $10.3892 / \mathrm{ol} .2016 .4269$

\begin{abstract}
Colorectal cancer (CRC) is a highly invasive tumor that is frequently associated with distant metastasis, which is the primary cause of poor prognosis. However, the mechanisms of metastasis remain poorly understood. MicroRNAs (miRNAs/miRs) have been considered to be implicated in CRC progression. In particular, miR-517a is proposed as a novel tumor-associated miRNA and has a potential role in tumor metastasis. The expression of miR-517a in CRC specimens was detected by reverse transcription-quantitative polymerase chain reaction. Transwell assays were performed to determine the migration and invasion of CRC cells. The putative target genes of miR-517a were disclosed using publicly available databases and western blot analysis. The present study identified that the expression of miR-517a was significantly higher in CRC tissues as compared with adjacent non-tumor tissues. Clinical analysis indicated that increased expression of miR-517a was correlated with poor prognostic features and poor long-term survival of CRC patients. In vitro evidences demonstrated that downregulation of miR-517a inhibited cell migration and invasion in HCT-116 cells. By contrast, upregulation of miR-517a increased the number of migrated and invaded SW480 cells. Notably, miR-517a expression was inversely regulated by forkhead box J3 (FOXJ3) abundance in CRC cells. Furthermore, an inverse correlation between miR-517a and FOXJ3 expression was observed in CRC tissues. In conclusion, miR-517a appears to be an independent prognostic marker for predicting survival of CRC patients, and may promote cell migration and invasion by inhibiting FOXJ3.
\end{abstract}

Correspondence to: Professor Qi Zhou, Department of Ultrasound, The Second Affiliated Hospital of Xi'an Jiaotong University, 157 West Fifth Road, Xi'an, Shaanxi 710004, P.R. China

E-mail: zq13909232905@163.com

Key words: miR-517a, colorectal cancer, prognostic marker, invasion, migration

\section{Introduction}

Colorectal cancer (CRC) is one of the most common type of cancer in the world, and its incidence is continuously increasing in China (1). Global cancer statistics indicate that CRC remains to be the fourth leading cause of cancer-associated mortalities worldwide, responsible for $>600,000$ mortalities annually (2). The major therapeutic approaches for CRC are surgery, neoadjuvant radiotherapy and adjuvant chemotherapy. Carcinoembryonic antigen and cancer antigen 19-9 are widely used biomarkers for CRC diagnosis. Endoscopy and blood screening have been proved to effectively reduce the incidence and mortality of CRC (3). However, the prognosis of CRC patients remains poor, with a 50-59\% 5-year survival rate (2).

MicroRNAs (miRNAs/miRs) are small non-coding RNA molecules that are 20-24 nucleotides in length, and regulate $60 \%$ of coding genes by preventing mRNA molecule translation and/or promoting degradation. Increasing evidence has revealed that miRNAs are critical in various diseases, including cancer. Novel functions and mechanisms by which miRNAs regulate genes are constantly being investigated (4-6).

miRNAs that promote carcinogenesis are termed oncomiRs (7). Numerous oncomiRs are overexpressed in CRC compared with noncancerous tissues, including miR-494, miR-21, miR-23a and miR-130b (8-11). These oncomiRs effect CRC cell proliferation, apoptosis, migration and invasion (12). However, another type of miRNA, termed tumor suppressive miRs (tsmiRs), are significantly downregulated in human colorectal adenocarcinoma tissue samples compared with the adjacent normal colorectal tissues, including miR-339-5p, miR-27a and miR-139-5p (13-15). miR-517a is considered to be a novel oncomiR and was observed to be elevated in human hepatocellular carcinoma (HCC) (16); expression of miR-517a increased proliferation, migration, and invasion of HCC cells in vitro (16). Furthermore, hsa-miR-517a exhibited significant inverse correlation with cyclin-dependent kinase $2 \mathrm{a}$ (CDKN2A) in glioblastoma; low expression of CDKN2A was associated with worse prognosis (17). A recent study reported that manipulation of miR-517a-3p expression changed lung cancer cell proliferation, migration and invasion capacity (18). Furthermore, miR-517a-3p accelerated lung cancer cell proliferation migration and invasion through inhibiting forkhead box J3 (FOXJ3) expression (18). However, the clinical significance of miR-517a in CRC, as well as its associated molecular 
pathways involved in the development and progression of $\mathrm{CRC}$, have yet to be elucidated.

The present study aimed to explore the clinical significance of miR-517a, and its role in CRC cell migration and invasion. The present study demonstrated that overexpression of miR-517a is observed in CRC tissues compared with noncancerous tissues, and its overexpression correlates with poor prognostic features. Furthermore, miR-517a appears to be an independent prognostic marker for predicting the survival of patients with CRC. In vitro experiments indicated that miR-517a promotes $\mathrm{CRC}$ cell migration and invasion. Mechanistically, the current results demonstrate that miR-517a may potentiate the invasive behavior of CRC cells by inhibiting FOXJ3.

\section{Materials and methods}

Clinical samples and cell lines. A total of 90 pairs of CRC and matched adjacent non-tumor tissue samples were obtained during colorectomies from the Department of Pathology, the Second Affiliated Hospital of Xi'an Jiaotong University (Xi'an, China) between January 2007 and January 2009. The clinical specimens were frozen and stored at $-80^{\circ} \mathrm{C}$ for reverse transcription-quantitative polymerase chain reaction (RT-qPCR) and western blot analysis. Samples were paraformaldehyde-fixed (Nanjing SenBeiJia Biological Technoogy Co., Ltd., Nanjing, China) and paraffin-embedded (Meryer Chemical Technology Co., Ltd., Shanghai, China). for immunohistochemical staining. The demographic features and clinicopathological parameters are indicated in Table I. All specimens had confirmed pathological diagnosis of CRC and were classified according to the International Union Against Cancer and American Joint Committee on Cancer criteria (7th edition) (19). Patients did not receive preoperative chemotherapy or embolization. All samples were used after obtaining informed consent. The Xi'an Jiaotong University Ethics Committee approved all protocols according to the Declaration of Helsinki (as revised in Tokyo 2004) (20).

In the present study, two CRC cell lines (HCT-116 and SW480) were used. In a pre-experiment, a noncancerous colon epithelial cell line (HCEC) was used as a control to evaluate the expression of miR-517a in HCT-116 and SW480 cells (data not shown). These cell lines were purchased from the Institute of Biochemistry and Cell Biology, Chinese Academy of Sciences, where they were established. Cells were cultured in complete Dulbecco's modified Eagle medium (DMEM; Gibco; Thermo Fisher Scientific, Inc., Waltham, MA, USA) containing $10 \%$ fetal bovine serum (FBS; Gibco; Thermo Fisher Scientific, Inc.) with $100 \mathrm{U} / \mathrm{ml}$ penicillin and $100 \mu \mathrm{g} / \mathrm{ml}$ streptomycin (Sigma-Aldrich, St. Louis, MO, USA) at $37^{\circ} \mathrm{C}$ in a humidified incubator containing $5 \% \mathrm{CO}_{2}$.

$R T$ - $q P C R$. The mir-Vana miRNA Isolation kit (Ambion; Thermo Fisher Scientific, Inc.) was used to isolate total RNA from frozen patient samples and cell lines, according to the manufacturer's protocol (21). The RNA was treated with DNase (Invitrogen; Thermo Fisher Scientific, Inc.). cDNA was synthesized using the Universal cDNA Synthesis kit II (Exiqon, Vedbaek, Denmark). The RT-qPCR analyses were performed in the ABI PRISM 7300 Sequence Detection System (Applied Biosystems; Thermo Fisher Scientific, Inc.) using ExiLENT SYBR Green Master Mix (Exiqon) for miR-517a. The reactions were incubated at $95^{\circ} \mathrm{C}$ for $60 \mathrm{sec}$, followed by 40 cycles of $95^{\circ} \mathrm{C}$ for $5 \mathrm{sec}$ and $60^{\circ} \mathrm{C}$ for $34 \mathrm{sec}$. RNU6B (U6) was measured as an internal control for miRNA. All samples were normalized to internal controls and fold changes were calculated based on relative quantification $\left(2^{-\Delta \Delta \mathrm{Cq}}\right)$. The primers for miR-517a and U6 were purchased from Exiqon. The following primer sequences were used: miR-517a stem-loop RT primer, CTCAACTGGTGTCGT GGAGTCGGCAATTCAGTTGAGACACTCTA; miR-517a forward, CGGCGGATCGTGCATCCCTTTA; miR-517a reverse, GTGCAGGGTCCGAGGT; U6 forward, CTCGCT TCGGCAGCACA; U6 reverse, AACGCTTCACGAATTTGC GT.

Target prediction. To determine the molecular mechanisms by which miR-517a inhibits CRC cell migration and invasion, predicted target genes of miR-517a were retrieved and analyzed using the publicly available databases, TargetScan 6.2 (www.targetscan.org) and miRanda (www.microrna.org).

Immunohistochemical staining. The paraffin-embedded tissue samples from postoperative patients were cut in 5-cm sections. Then the samples were deparaffinized in xylene (ZSGB-BIO, Beijing, China) and rehydrated using a series of graded alcohol. Slides were blocked with $10 \%$ goat serum (ZSGB-BIO) prior to incubation with rabbit anti-human polyclonal FOXJ3 (dilution, 1:100; cat no. SAB4500907; Sigma-Aldrich) antibody. The samples were incubated overnight with the primary antibody at $4^{\circ} \mathrm{C}$, and then incubated with goat anti-rabbit secondary antibody (dilution, 1:1,000; cat no. ZDR-5403; ZSGB-BIO) at room temperature for $2 \mathrm{~h}$ followed by 3,3'-diaminobenzidine-labeled secondary antibody. Subsequently, sections were counterstained with hematoxylin (ZSGB-BIO). Protein staining was evaluated under a light microscope (BX-51; Olympus, Tokyo, Japan) at x400 magnification.

Cell transfection. miRNA vectors, including miR-517a expression vector (HmiR0330-MR04), scrambled control vector for miR-517a (CmiR0001-MR04), miR-517a inhibitor (HmiR-AN0575-AM04) and negative control for the miR-517a inhibitor (CmiR-AN0001-AM04), were purchased from Genecopoeia (Guangzhou, China). Cells were transfected with the aforementioned vectors using Lipofectamine 2000, according to the manufacturer's instructions (Invitrogen; Thermo Fisher Scientific, Inc.).

Transwell assays. Transwell migration assays were performed in 12-well plates with $8-\mu \mathrm{m}$ BioCoat control inserts (BD Biosciences, Bedford, MA, USA). Cells $\left(1-2 \times 10^{5}\right)$ that were suspended in $500 \mu 1$ serum-free DMEM were seeded in the upper well. DMEM medium with $10 \%$ FBS was added to the lower well. After $12 \mathrm{~h}$ incubation, the membranes were removed, cells on the side facing the upper well were wiped with a cotton swab, and the membranes were stained with crystal violet (MCE China, Shanghai, China). At least 6 representative images of each well were captured (BX-51; Olympus, Tokyo, Japan) and cell numbers were counted using ImageJ software (version 1.46r; National Institutes of Health, Bethesda, MD, USA). A BioCoat Matrigel invasion chamber 
Table I. Clinical association analysis of miR-517a expression in patients with colorectal cancer.

\begin{tabular}{|c|c|c|c|c|}
\hline \multirow[b]{2}{*}{ Feature } & \multirow[b]{2}{*}{ Total patients, $\mathrm{n}(\mathrm{n}=90)$} & \multicolumn{2}{|c|}{ Patients, $\mathrm{n}$} & \multirow[b]{2}{*}{ P-value } \\
\hline & & Low miR-517a & High miR-517a & \\
\hline Age, years & & & & 0.490 \\
\hline$\leq 60$ & 27 & 15 & 12 & \\
\hline$>60$ & 63 & 30 & 33 & \\
\hline Gender & & & & 0.389 \\
\hline Male & 54 & 29 & 25 & \\
\hline Female & 36 & 16 & 20 & \\
\hline Tumor grade & & & & 0.468 \\
\hline $\mathrm{G} 1 / \mathrm{G} 2$ & 67 & 35 & 32 & \\
\hline G3/G4 & 23 & 10 & 13 & \\
\hline Size, cm & & & & 0.660 \\
\hline$<5$ & 32 & 17 & 15 & \\
\hline$\geq 5$ & 58 & 28 & 30 & \\
\hline Tumor invasion & & & & $0.011^{\mathrm{a}}$ \\
\hline $\mathrm{T} 1 / \mathrm{T} 2$ & 20 & 15 & 5 & \\
\hline $\mathrm{T} 3 / \mathrm{T} 4$ & 70 & 30 & 40 & \\
\hline Lymph node metastases & & & & $0.038^{\mathrm{a}}$ \\
\hline Absent & 63 & 36 & 27 & \\
\hline Present & 27 & 9 & 18 & \\
\hline Distant metastasis & & & & $0.020^{\mathrm{a}}$ \\
\hline Absent & 71 & 40 & 31 & \\
\hline Present & 19 & 5 & 14 & \\
\hline TNM stage & & & & $0.001^{\mathrm{a}}$ \\
\hline $\mathrm{I} / \mathrm{II}$ & 54 & 35 & 19 & \\
\hline III/IV & 36 & 10 & 26 & \\
\hline
\end{tabular}

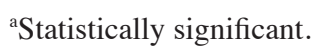

(BD Biosciences) was used for Transwell invasion assays; otherwise, the same protocols were used for Transwell migration assays. The experiments were performed in triplicate.

Western blot analysis. Total proteins were extracted from cells with radioimmunoprecipitation lysis buffer (Santa Cruz Biotechnology, Inc., Santa Cruz, CA, USA) and centrifuged at $14,000 \mathrm{x} \mathrm{g}$ for $10 \mathrm{~min}$, separated by $10 \%$ sodium dodecyl sulfate-polyacrylamide gel electrophoresis (Sigma-Aldrich) at $165 \mathrm{~V}$ for $1.5 \mathrm{~h}$ and then transferred onto a PVDF membrane (Roche, Indianapolis, IN, USA). The membrane was blocked with 5\% skimmed milk (Shanghai Haoran Bio Technologies Co., Ltd., Shanghai, China and incubated with the appropriate antibody overnight at $4^{\circ} \mathrm{C}$. The following primary antibodies were used: Rabbit anti-human polyclonal FOXJ3 (dilution, 1:1,000; cat no. SAB4500907; Sigma-Aldrich) and mouse anti-human monoclonal GAPDH (dilution, 1:5,000; cat no. G8140; United States Biological, Swampscott, MA, USA). Horseradish peroxidase-conjugated donkey anti-rabbit and goat anti-mouse secondary antibodies (cat nos. sc-2313 and sc-2005, respectively; Santa Cruz Biotechnology, Inc.) were used at room temperature for $2 \mathrm{~h}$ with a dilution of
1:1,000-1:5,000 and expression as detected using enhanced chemiluminescence reagents (Thermo Fisher Scientific, Inc.).

Statistical analysis. Results are expressed as the mean \pm standard error of the mean. The quantitative data were compared between two groups using the two-tailed Student's t-test. Categorical data were analyzed using the Pearson's $\chi^{2}$ test. The Kaplan-Meier method and log-rank test were used to compare the cumulative recurrence and survival rates. The independent factors influencing the survival and recurrence of CRC patients were determined using the Cox proportional hazards model. Correlation analysis was tested by the Spearman's rank correlation coefficient. $\mathrm{P}<0.05$ was considered to indicate statistically significant differences.

\section{Results}

Clinical significance of miR-517a in CRC specimens. The expression of miR-517a was determined by RT-qPCR and normalized against an endogenous control (U6 RNA) in 90 pairs of CRC and adjacent non-tumor tissues. The data indicated that the expression of miR-517a in CRC tissues was significantly 
Table II. Multivariate Cox regression analysis of 5-year overall and disease-free survival of 90 patients with colorectal cancer.

\begin{tabular}{|c|c|c|c|c|c|c|}
\hline \multirow[b]{2}{*}{ Variable } & \multicolumn{3}{|c|}{ Overall survival } & \multicolumn{3}{|c|}{ Disease-free survival } \\
\hline & HR & $95 \% \mathrm{CI}$ & P-value & HR & $95 \% \mathrm{CI}$ & P-value \\
\hline Tumor invasion (T1/T2 vs. T3/T4) & 1.511 & $0.998-2.410$ & 0.051 & 1.523 & $0.969-2.395$ & 0.068 \\
\hline Lymph node metastases (absent vs. present) & 1.437 & $0.939-2.200$ & 0.095 & 1.395 & $0.894-2.178$ & 0.143 \\
\hline Distant metastasis (absent vs. present) & 1.928 & $1.161-3.204$ & $0.011^{\mathrm{a}}$ & 1.385 & $0.911-2.106$ & 0.127 \\
\hline TNM stage (I/II vs. III/IV) & 1.993 & $1.290-3.080$ & $0.002^{\mathrm{a}}$ & 1.623 & $1.034-2.548$ & $0.035^{\mathrm{a}}$ \\
\hline miR-517a expression (low vs. high) & 2.394 & $1.466-3.909$ & $<0.001^{\mathrm{a}}$ & 1.754 & $1.033-2.978$ & $0.038^{\mathrm{a}}$ \\
\hline
\end{tabular}

${ }^{a}$ Statistically significant. HR, hazard ratio; CI, confidence interval.

A

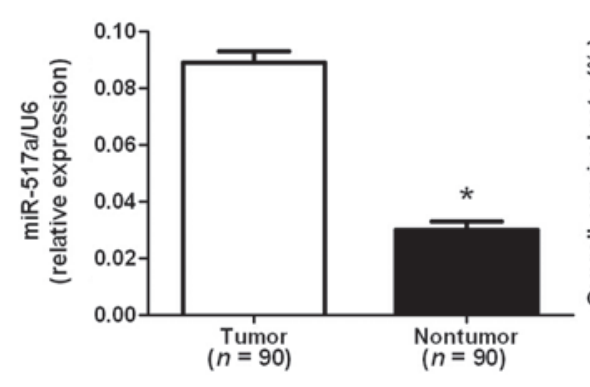

B

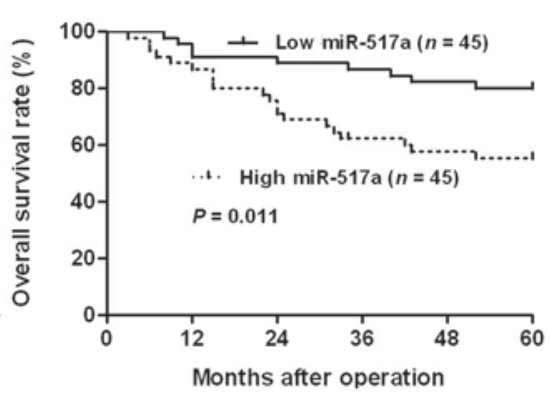

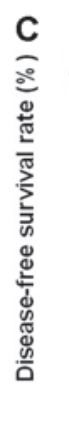

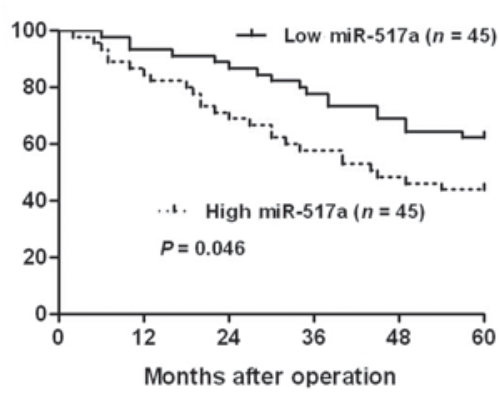

Figure 1. Expression and prognostic significance of miR-517a in colorectal cancer (CRC). (A) Comparing differences in the expression levels of miR-517a between $\mathrm{CRC}$ tissues (tumor) and matched adjacent non-tumor tissues. ${ }^{\mathrm{P}}<0.05$ vs. tumor. According to the level of miR-517a expression, Kaplan-Meier 5-year (B) overall and (C) disease-free survival curves of CRC patients showed that high expression of miR-517a was correlated with poor prognosis. The median miR-517a expression value obtained for the $90 \mathrm{CRC}$ samples, as detected by reverse transcription-quantitative polymerase chain reaction, was used as the cutoff value. miR, micro RNA.

higher than in adjacent non-tumor tissues $(\mathrm{P}<0.001$; Fig. 1A). The expression of miR-517a was considered to be low $(n=45)$ or high $(\mathrm{n}=45)$ according to the cutoff value, which was defined as the median of the cohort. As indicated in Table I, clinical association analysis revealed that high expression of miR-517a was prominently associated with poor prognostic features, including high tumor invasion, lymph node metastases, distant metastasis and advanced TNM stage $(\mathrm{P}<0.05)$. Furthermore, $\mathrm{CRC}$ patients with high expression of $\mathrm{miR}-517 \mathrm{a}$ were associated with poor overall and disease-free survival $(\mathrm{P}=0.011$ and 0.046 , respectively; Fig. 1B and C). Notably, miR-517a expression, in addition to TNM stage, is an independent factor for predicting the survival of patients with $\mathrm{CRC}(\mathrm{P}<0.05$; Table II). These data indicate that miR-517a acts as a potent biomarker for predicting the prognosis of patients with CRC.

miR-517a promotes cell migration and invasion in CRC. A noncancerous colon epithelial cell line (HCEC) was used as a control to evaluate the expression of miR-517a in HCT-116 and SW480 cells in a pre-experiment. The levels of miR-517a expression in the HCT-116 and SW480 cells were approximately 4.6- and-1.5 fold higher compared with the HCEC cells (data not shown). To investigate the potential role of miR-517a in $\mathrm{CRC}$, the expression of miR-517a was altered by transfecting CRC cells with miR-517a mimics and inhibitors. As assessed by RT-qPCR, the expression of miR-517a was significantly downregulated by miR-517a inhibitors in HCT-116 cells $(\mathrm{P}=0.011$;
Fig. 2A). Transwell migration assays were performed to analyze the effect of altering miR-517a levels on cell migration. It was determined that downregulation of miR-517a led to a significant reduction of cell migration in $\mathrm{HCT}-116$ cells $(\mathrm{P}=0.018$; Fig. $2 \mathrm{~B})$. Furthermore, as determined by Transwell invasion assays, the number of invaded HCT-116 cells was significantly reduced following downregulation of miR-517a ( $\mathrm{P}=0.032$; Fig. 2B). Next, SW480 cells, which showed a significant 5.43 -fold reduction in miR-517a expression compared with HCT-116 cells under normal conditions, were used for gain-of-function experiments. miR-517a-overexpressing SW480 cells were established and confirmed by RT-qPCR ( $\mathrm{P}=0.005$; Fig. 3A). As expected, upregulation of miR-517a significantly increased the number of migrated and invaded SW480 cells $(\mathrm{P}=0.008$ and 0.019 , respectively; Fig. 3B). Thus, miR-517a appears to promote cell migration and invasion in CRC.

miR-517a inversely regulates FOXJ3 abundance in CRC. A previous study reported that miR-517a-3p accelerates cell proliferation, migration and invasion through inhibiting FOXJ3 expression in human lung cancer (18). In addition, TargetScan and miRanda databases were used to identify FOXJ3 as one of the targets of miR-517a. Therefore, HCT-116 cells transfected with negative control or miR-517a inhibitors were subjected to western blotting for FOXJ3. As assessed by immunoblotting, downregulation of miR-517a significantly increased the expression of FOXJ3 protein in HCT-116 cells ( $\mathrm{P}=0.014$; Fig. 4A). 
A

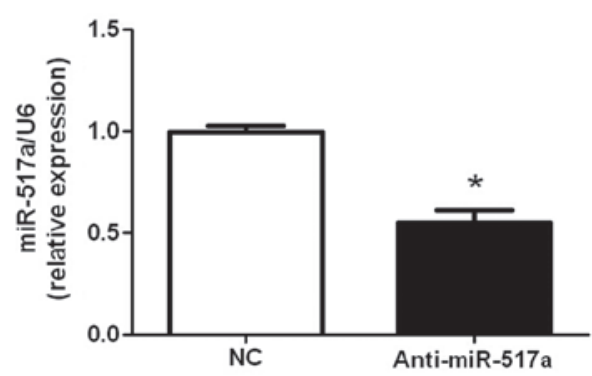

B

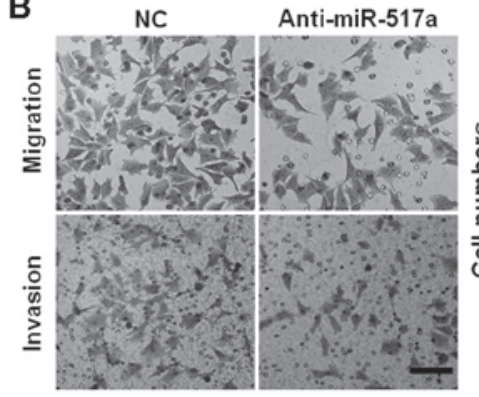

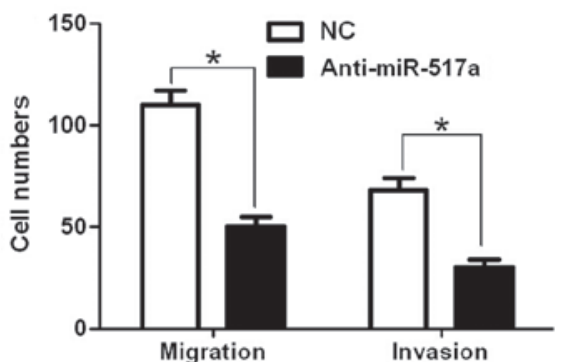

Figure 2. Downregulation of miR-517a inhibits HCT-116 cell migration and invasion. (A) HCT-116 cells that were transfected with NC and miR-517a inhibitor (anti-miR-517a) were subjected to reverse transcription-quantitative polymerase chain reaction to determine miR-517a expression. $\mathrm{n}=3$ independent experiments. "P<0.05 vs. NC. (B) Cell migration and invasion, as measured by Transwell assays, were inhibited by downregulation of miR-517a in HCT-116 cells as compared with control cells. $\mathrm{n}=3$ repeats with similar results. ${ }^{*} \mathrm{P}<0.05$. Scale bar, $100 \mu \mathrm{m}$. Crystal violet staining. miR, micro RNA; NC, negative control.

A

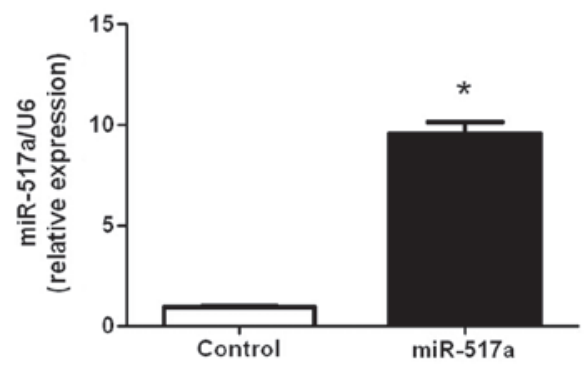

B

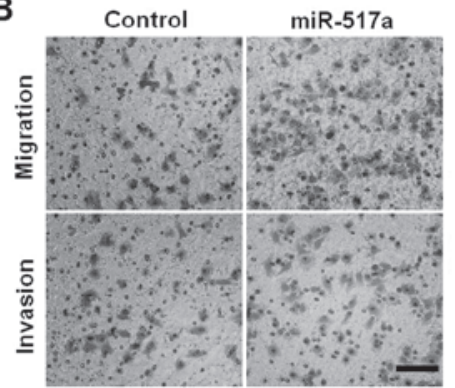

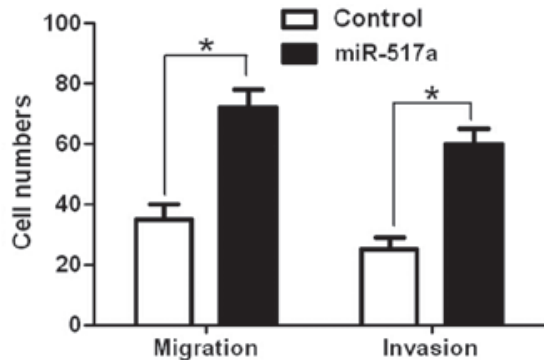

Figure 3. Upregulation of miR-517a inhibits SW480 cell migration and invasion. (A) SW480 cells that were transfected with miR-control (control) and miR-517a were subjected to reverse transcription-quantitative polymerase chain reaction to determine miR-517a expression. $n=3$ independent experiments. ${ }^{*} \mathrm{P}<0.05$ vs. control. (B) miR-517a-overexpressing SW480 cells conferred greater cell migration and invasion as compared with control cells. $\mathrm{n}=3$ repeats with similar results. " $\mathrm{P}<0.05$. Scale bar, $100 \mu \mathrm{m}$. Crystal violet staining. miR, micro RNA.
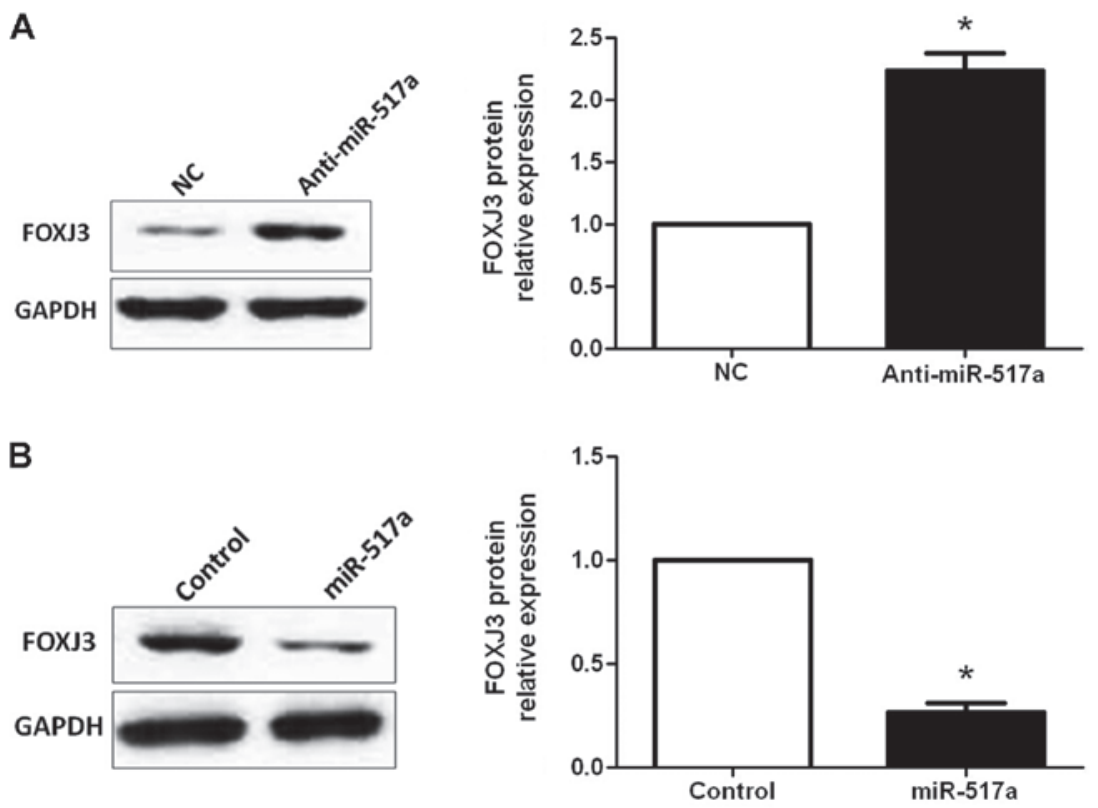

Figure 4. miR-517a inversely regulates FOXJ3 expression in colorectal cancer cells. (A) Downregulation of miR-517a increased FOXJ3 protein in HCT-116 cells. $\mathrm{n}=3$ independent experiments, ${ }^{*} \mathrm{P}<0.05$. (B) Upregulation of miR-517a led to a significant reduction of FOXJ3 expression in SW480 cells. $\mathrm{n}=3$ independent experiments, ${ }^{\mathrm{P}}<0.05$. NC, negative control; FOXJ3, forkhead box J3; miR, micro RNA.

By contrast, upregulation of miR-517a significantly decreased the expression of FOXJ3 protein in SW480 cells $(\mathrm{P}=0.004$; Fig. 4B). Next, the correlation between FOXJ3 and miR-517a expression were evaluated in CRC tissues. The expression of FOXJ3 in miR-517a high-expressing tumors was significantly lower than those in miR-517a low-expressing tumors 


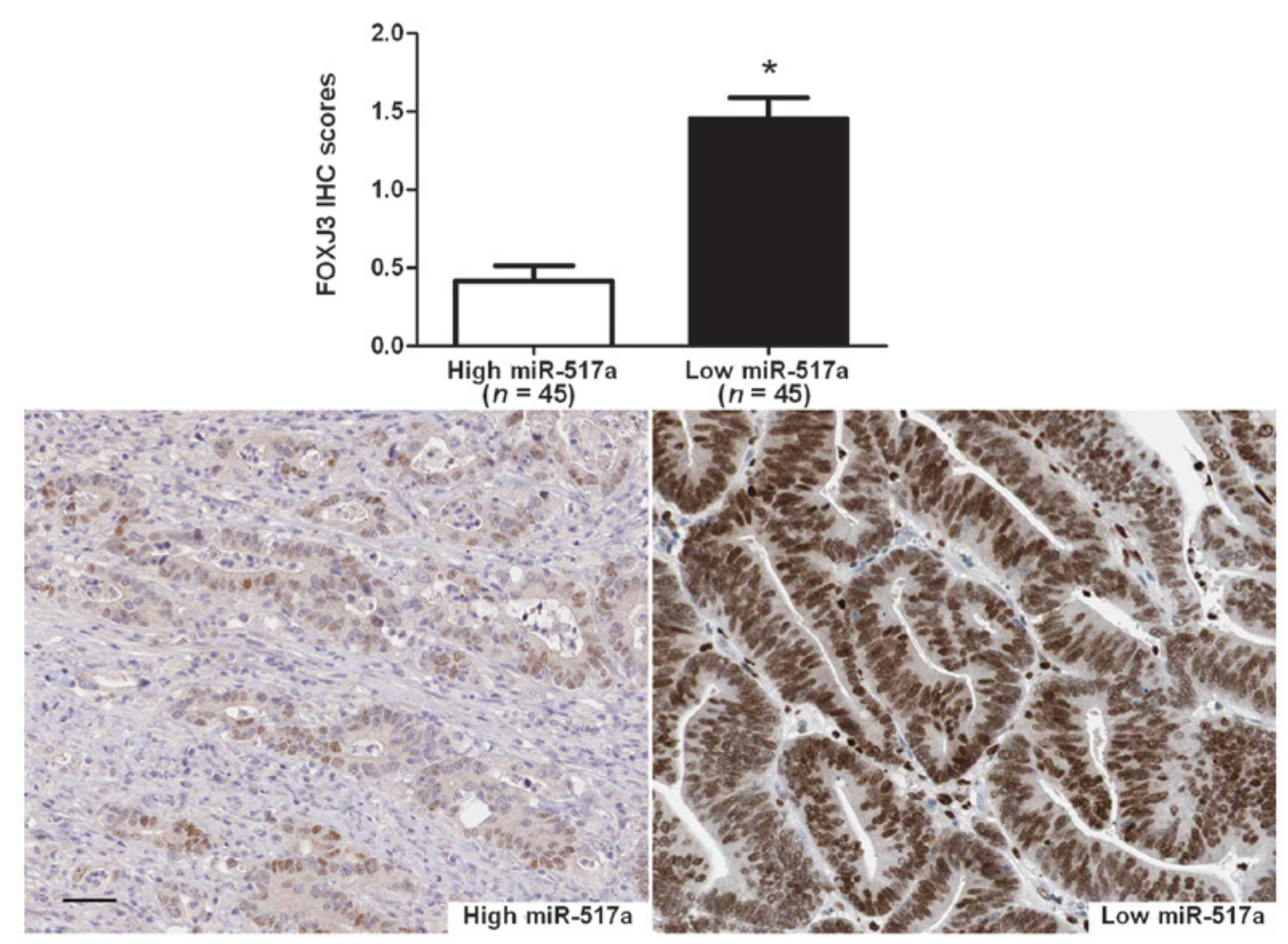

Figure 5. An inverse correlation between miR-517a and FOXJ3 is observed in colorectal cancer tissues. In cases of low miR-517a expression, there was strong FOXJ3 protein expression in the same tissue section. By contrast, in the case of high miR-517a expression, there was weak FOXJ3 protein expression. Quantitative data showed that the expression of FOXJ3 in miR-517a high-expressing tumors was significantly lower than those in miR-517a low-expressing tumors. Scale bar, $50 \mu \mathrm{m}$. 3,3'-diaminobenzidine staining. "P<0.05. FOXJ3, forkhead box J3; IHC, immunohistochemistry; miR, micro RNA.

$(\mathrm{P}<0.001$; Fig. 5). Furthermore, Spearman's rank correlation analysis indicated that miR-517a was inversely correlated with FOXJ3 expression in CRC tissues $(\mathrm{r}=-0.458 ; \mathrm{P}=0.012)$. Taken together, these data indicate that miR-517a suppresses FOXJ3 expression in CRC.

\section{Discussion}

Increasing evidence has indicated that the deregulation and dysfunction of miRNAs is key in the development and progression of CRC $(22,23)$. Increased expression of oncomiRs and decreased expression of tsmiRs have been observed in CRC tissues as compared with adjacent non-tumor tissues (24). However, miRNAs that are correlated with the recurrence and metastasis of CRC remain poorly understood. Considering the findings of previous studies, the present study aimed to explore the clinical significance of miR-517a and its role in CRC cell migration and invasion. The current data demonstrated that the expression of miR-517a in CRC tissues was significantly higher than in adjacent noncancerous tissues. Clinical analysis determined that high expression of miR-517a was significantly correlated with high tumor invasion, lymph node metastases, distant metastasis and advanced TNM stage in CRC. Furthermore, Kaplan-Meier and multivariate Cox regression analysis demonstrated that miR-517a was an independent prognostic marker for the predicting survival of patients with CRC. Together, these results suggest that miR-517a is critical for the prognostic determination in CRC.

miR-517a has been proposed to be involved in tumor metastasis of HCC and lung cancer $(16,18)$. Therefore, the role of
miR-517a in CRC was further investigated. The present study identified that downregulation of miR-517a inhibited cell migration and invasion in HCT-116 cells. Furthermore, upregulation of miR-517a increased the number of migrated and invaded SW480 cells. These data suggest that miR-517a promotes cell migration and invasion in CRC. To determine the molecular mechanisms by which miR-517a inhibits CRC cell migration and invasion, predicted target genes of miR-517a were retrieved and analyzed using publicly available databases (TargetScan and miRanda). FOXJ3, which is known to be a key transcription factor of mitochondrial biogenesis and was identified to upregulate myocyte enhancer factor $2 \mathrm{C}(25,26)$, was predicted as one of the targets of miR-517a. A recent study reported that miR-517a-3p accelerates cell proliferation, migration and invasion through inhibiting FOXJ3 expression in human lung cancer (18). Thus, the regulatory effect of miR-517a on FOXJ3 in CRC cells was investigated. The data indicated that downregulation of miR-517a increased the expression level of FOXJ3 protein in HCT-116 cells. By contrast, upregulation of miR-517a reduced FOXJ3 expression in SW480 cells. Furthermore, a significant inverse correlation between miR-517a and FOXJ3 expression was observed in CRC tissues. Accordingly, we propose that miR-517a may promote cell migration and invasion by targeting FOXJ3 in CRC.

In conclusion, the present study determined that miR-517a is overexpressed in CRC and its high expression is associated with poor prognostic features. Furthermore, high expression of miR-517a appears to be a prognostic marker for predicting poor survival of CRC patients. In vitro experiments demonstrated that miR-517a facilitates CRC cell migration and invasion. 
Mechanistically, we propose that miR-517a may promote CRC cell mobility by suppressing FOXJ3. Taken together, we propose that miR-517a may potentially act as a clinical biomarker and may also be a therapeutic target in CRC.

\section{Acknowledgements}

The present study was supported by a grant from the National Natural Science Foundation of China (no. 81171356).

\section{References}

1. Li L and Ma BB: Colorectal cancer in Chinese patients: Current and emerging treatment options. Onco Targets Ther 7: 1817-1828, 2014.

2. Jemal A, Bray F, Center MM, Ferlay J, Ward E and Forman D: Global cancer statistics. CA Cancer J Clin 61: 69-90, 2011.

3. van Hees F, Saini SD, Lansdorp-Vogelaar I, Vijan S, Meester RG de Koning HJ, Zauber AG and van Ballegooijen M: Personalizing colonoscopy screening for elderly individuals based on screening history, cancer risk, and comorbidity status could increase cost effectiveness. Gastroenterology 149: 1425-1437, 2015.

4. Croce CM: Causes and consequences of microRNA dysregulation in cancer. Nat Rev Genet 10: 704-714, 2009.

5. Wu WK, Lee CW, Cho CH, Fan D, Wu K, Yu J and Sung JJ: MicroRNA dysregulation in gastric cancer: A new player enters the game. Oncogene 29: 5761-5771, 2010.

6. Tu K, Zheng X, Dou C, Li C, Yang W, Yao Y and Liu Q: MicroRNA-130b promotes cell aggressiveness by inhibiting peroxisome proliferator-activated receptor gamma in human hepatocellular carcinoma. Int J Mol Sci 15: 20486-20499, 2014.

7. Tu K, Li C, Zheng X, Yang W, Yao Y and Liu Q: Prognostic significance of miR-218 in human hepatocellular carcinoma and its role in cell growth. Oncol Rep 32: 1571-1577, 2014.

8. Sun HB, Chen X, Ji H, Wu T, Lu HW, Zhang Y, Li H and Li YM: miR494 is an independent prognostic factor and promotes cell migration and invasion in colorectal cancer by directly targeting PTEN. Int J Oncol 45: 2486-2494, 2014.

9. Liu K, Li G, Fan C, Zhou X, Wu B and Li J: Increased expression of microRNA-21 and its association with chemotherapeutic response in human colorectal cancer. J Int Med Res 39: 2288-2295, 2011.

10. Jahid S, Sun J, Edwards RA, Dizon D, Panarelli NC, Milsom JW, Sikandar SS, Gümüs ZH and Lipkin SM: miR-23a promotes the transition from indolent to invasive colorectal cancer. Cancer Discov 2: 540-553, 2012.

11. Colangelo T, Fucci A, Votino C, Sabatino L, Pancione M, Laudanna C, Binaschi M, Bigioni M, Maggi CA, Parente D, et al: MicroRNA-130b promotes tumor development and is associated with poor prognosis in colorectal cancer. Neoplasia 15: 1218-1231, 2013
12. Ahmed FE: miRNA as markers for the diagnostic screening of colon cancer. Expert Rev Anticancer Ther 14: 463-485, 2014.

13. Zhang C, Liu J, Wang X, Wu R, Lin M, Laddha SV, Yang Q, Chan CS and Feng Z: MicroRNA-339-5p inhibits colorectal tumorigenesis through regulation of the MDM2/p53 signaling. Oncotarget 5: 9106-9117, 2014.

14. Bao Y, Chen Z, Guo Y, Feng Y, Li Z, Han W, Wang J, Zhao W, Jiao Y, Li K, et al: Tumor suppressor microRNA-27a in colorectal carcinogenesis and progression by targeting SGPP1 and Smad2. PLoS One 9: e105991, 2014.

15. Zhang L, Dong Y, Zhu N, Tsoi H, Zhao Z, Wu CW, Wang K, Zheng S, Ng SS, Chan FK, et al: microRNA-139-5p exerts tumor suppressor function by targeting NOTCH1 in colorectal cancer. Mol Cancer 13: 124, 2014

16. Toffanin S, Hoshida Y,Lachenmayer A, Villanueva A, Cabellos L, Minguez B, Savic R, Ward SC, Thung S, Chiang DY, et al: MicroRNA-based classification of hepatocellular carcinoma and oncogenic role of miR-517a. Gastroenterology 140: 1618-1628, e16, 2011

17. Feng J, Kim ST, Liu W, Zhang Z, Zhu Y, Berens M, Sun J and $\mathrm{Xu} \mathrm{J}$ : An integrated analysis of germline and somatic, genetic and epigenetic alterations at 9p21.3 in glioblastoma. Cancer 118: 232-240, 2012.

18. Jin J, Zhou S, Li C, Xu R, Zu L, You J and Zhang B: MiR-517a-3p accelerates lung cancer cell proliferation and invasion through inhibiting FOXJ3 expression. Life Sci 108: 48-53, 2014.

19. Tong LL, Gao P, Wang ZN, Song YX, Xu YY, Sun Z, Xing CZ and Xu HM: Is the seventh edition of the UICC/AJCC TNM staging system reasonable for patients with tumor deposits in colorectal cancer? Ann Surg 255: 208-213, 2012.

20. World Medical Association: World Medical Association Declaration of Helsinki: Ethical principles for medical research involving human subjects. JAMA 310: 2191-2194, 2013.

21. Chang RM, Yang H, Fang F, Xu JF and Yang LY: MicroRNA-331-3p promotes proliferation and metastasis of hepatocellular carcinoma by targeting $\mathrm{PH}$ domain and leucine-rich repeat protein phosphatase. Hepatology 60: 1251-1263, 2014.

22. Muhammad S, Kaur K, Huang R, Zhang Q, Kaur P, Yazdani HO, Bilal MU, Zheng J, Zheng L and Wang XS: MicroRNAs in colorectal cancer: Role in metastasis and clinical perspectives. World J Gastroenterol 20: 17011-17019, 2014

23. Tokarz $\mathrm{P}$ and Blasiak J: The role of microRNA in metastatic colorectal cancer and its significance in cancer prognosis and treatment. Acta Biochim Pol 59: 467-474, 2012.

24. Bonfrate L, Altomare DF, Di Lena M, Travaglio E, Rotelli MT, De Luca A and Portincasa P: MicroRNA in colorectal cancer: New perspectives for diagnosis, prognosis and treatment. J Gastrointestin Liver Dis 22: 311-320, 2013.

25. Landgren $\mathrm{H}$ and Carlsson P: FoxJ3, a novel mammalian forkhead gene expressed in neuroectoderm, neural crest and myotome. Dev Dyn 231: 396-401, 2004.

26. Alexander MS, Shi X, Voelker KA, Grange RW, Garcia JA, Hammer RE and Garry DJ: Foxj3 transcriptionally activates Mef2c and regulates adult skeletal muscle fiber type identity. Dev Biol 337: 396-404, 2010. 